\title{
Prolongation of the QT interval by ketanserin
}

\author{
H.A. Cameron, ${ }^{*}$ P.C. Waller, $\dagger$ and L.E. Ramsay \\ University Department of Therapeutics, Royal Hallamshire Hospital, Sheffield S10 2JF, UK.
}

\begin{abstract}
Summary: In hypertensive patients single doses of ketanserin $40 \mathrm{mg}$ prolonged the corrected QT interval (QT $)$ for at least 8 hours, with a maximal increase of $35 \mathrm{~ms}(P<0.001, n=6)$ after 2 hours. During chronic dosing (20 and $40 \mathrm{mg}$ b.d.) the $Q T_{c}$ was further prolonged, by 46 and $45 \mathrm{~ms}$ respectively. $Q T_{c}$ prolongation after treatment with a mean dose of $73 \mathrm{mg} /$ day for 7 weeks $(n=26)$ was significantly related to body weight $(r=-0.58, P<0.01)$, and to the dose of ketanserin corrected for body weight $(r=0.63, P<0.01)$, but not to plasma concentrations of ketanserin, ketanserinol, potassium or calcium. High doses of ketanserin (mean dose $167 \mathrm{mg} / \mathrm{day}, n=9$ ) increased the $Q T_{c}$ by $40 \mathrm{~ms}(P<0.001)$, with prolongation of up to $80 \mathrm{~ms}$ in individual patients. Treatment with ketanserin at doses proposed for clinical use $(40-80 \mathrm{mg} / \mathrm{day})$ may carry a risk of ventricular arrhythmias.
\end{abstract}

\section{Introduction}

Ketanserin is a selective antagonist of serotonin (5hydroxytryptamine, 5-HT) at 5- $\mathrm{HT}_{2}$ receptors which is currently being evaluated for the treatment of hypertension, ${ }^{1,2}$ intermittent claudication ${ }^{3,4}$ and Raynaud's phenomenon. ${ }^{5}$ It prolongs the corrected QT interval on the electrocardiogram (ECG) of healthy subjects. ${ }^{6}$ Drug-induced QT prolongation may predispose to serious ventricular arrhythmias, particularly the polymorphous form of ventricular tachycardia known as torsade de pointes. ${ }^{7}$ We have examined the influence of ketanserin on the QT interval of 81 patients with hypertension or intermittent claudication who participated in three studies. Our aims were to confirm that ketanserin prolonged the QT interval; to examine the timecourse of the phenomenon after single doses and during chronic treatment; to determine whether the effect was dose-dependent; and to explore the relation of QT prolongation to plasma drug concentration and other variables such as body weight.

\section{Materials and methods}

All patients gave written informed consent to the studies which were approved by the hospital ethics

Correspondence: L.E. Ramsay, F.R.C.P.

Present addresses: *I.C.I. Pharmaceuticals Division, Mereside, Alderley Park, Macclesfield, Cheshire SK10 4TG, UK. †Glasgow Blood Pressure Clinic, Western Infirmary, Glasgow G11 6NT, UK.

Accepted: 3 September 1987 committee. The protocols are described in the results section for clarity of presentation. No patient was taking any drug which causes pronounced prolongation of the QT interval, and drug therapy other than ketanserin or placebo was held constant throughout all three studies. Patients with important hepatic or renal dysfunction were excluded.

In each patient the QT interval was measured from a single lead (usually lead II), recorded at a paper speed of $50 \mathrm{~mm} / \mathrm{s}$, as the time from the first deflection of the QRS complex to the point where a tangent drawn along the descending limb of the $T$ wave crossed the isoelectric line. $U$ waves were excluded. All measurements were made by a single observer who was unaware of the treatment taken or sequence of traces, to avoid bias. The QT interval was corrected for heart rate using Bazett's correction. ${ }^{8}$ Because of controversy over the most appropriate method of correcting the QT interval for heart rate $^{9}$ a further method of analysis was used in Study 2, the largest of the three studies. The relations between the uncorrected QT interval and the $\mathbf{R}-\mathbf{R}$ interval were compared in ketanserin and placebo-treated patients by linear regression. The slopes of the regression lines were constrained to be parallel $^{10}$ and the intercepts for ketanserin and placebo treatment were compared. A significant difference between the intercepts in this analysis indicates a difference in QT interval which is independent of heart rate and any correction factor.

Blood was taken for assay of plasma concentrations of ketanserin and its major metabolite ketanserinol, at the time of each QT interval

(C) The Fellowship of Postgraduate Medicine, 1988 
measurement. Samples were stored at $-20^{\circ} \mathrm{C}$ prior to assay by high performance liquid chromatography with fluorescence detection. R 46594 (Janssen Pharmaceutica, Beerse) was used as an internal standard. The limit of detection of the assay was $1 \mathrm{ng} / \mathrm{ml}$ and the coefficients of variation were $5.0 \%$ (ketanserin) and $2.7 \%$ (ketanserinol) at a concentration of $200 \mathrm{ng} / \mathrm{ml}$. Serum potassium and calcium concentrations were measured before treatment and at the final assessment. Serum calcium values were corrected for serum albumin concentration.

Histograms and normal probability plots suggested that the data were normally distributed. Values for plasma ketanserin and ketanserinol concentrations were log-transformed before analysis. The statistical methods used were paired $t$-tests, analysis of variance and multiple linear regression.

\section{Results}

\section{Study 1}

Protocol Six patients with essential hypertension ( 3 men, 3 women; mean age 59.8 years) were given a single oral dose of $40 \mathrm{mg}$ ketanserin and changes in the $\mathrm{QT}_{\mathrm{c}}$ interval were assessed over the next 8 hours. The patients were then treated with ketanserin $20 \mathrm{mg}$ b.d. for 4 weeks, and changes in the $\mathrm{QT}_{\mathrm{c}}$ interval were examined for 4 hours following the morning dose. They were subsequently treated with ketanserin $40 \mathrm{mg}$ b.d. for 4 weeks and the same procedure was repeated.

Results The changes in $\mathrm{QT}_{c}$ and mean plasma drug concentrations after a single $40 \mathrm{mg}$ dose of ketanserin are shown in Figure 1. The $\mathrm{QT}_{\mathrm{c}}$ averaged $401 \mathrm{~ms}$ before dosing, increased by $26 \mathrm{~ms}(P<0.01)$ after 1 hour, reached a maximum increase of $35 \mathrm{~ms} \quad(P<0.001)$ at 2 hours, and remained significantly prolonged after 4 and 8 hours. Plasma concentrations of ketanserin and ketanserinol peaked after 1 and 2 hours respectively and paralleled the increased in $\mathrm{QT}_{\mathrm{c}}$ interval (Figure 1). The findings at steady-state after four weeks of treatment are shown in Figure 2. During treatment with ketanserin $20 \mathrm{mg}$ b.d. the $\mathrm{QT}_{\mathrm{c}}$ was increased by $19 \mathrm{~ms}$ (not significant) before the morning dose, and lengthened to a maximum increase of $46 \mathrm{~ms}$ $(P<0.01)$ four hours after dosing. After four weeks' treatment with ketanserin $40 \mathrm{mg}$ b.d. $\mathrm{QT}_{\mathrm{c}}$ prolongation before dosing was $24 \mathrm{~ms}$ (not significant), increasing further to $45 \mathrm{~ms}(P<0.01)$ at 4 hours. Change in $\mathrm{QT}_{\mathrm{c}}$ interval was weakly but significantly correlated with plasma ketanserin concentration $(P=0.48,95 \%$ CI $0.21,0.75)$ and plasma ketanserinol concentration $(P=0.54,95 \%$ CI 0.27, 0.81).

Comment Single doses of ketanserin increased the $\mathrm{QT}_{\mathrm{c}}$ interval substantially and the effect was more pronounced during chronic dosing. At steady-state the degree of lengthening varied markedly during the dose interval, with a two fold difference in $\mathrm{QT}_{\mathrm{c}}$
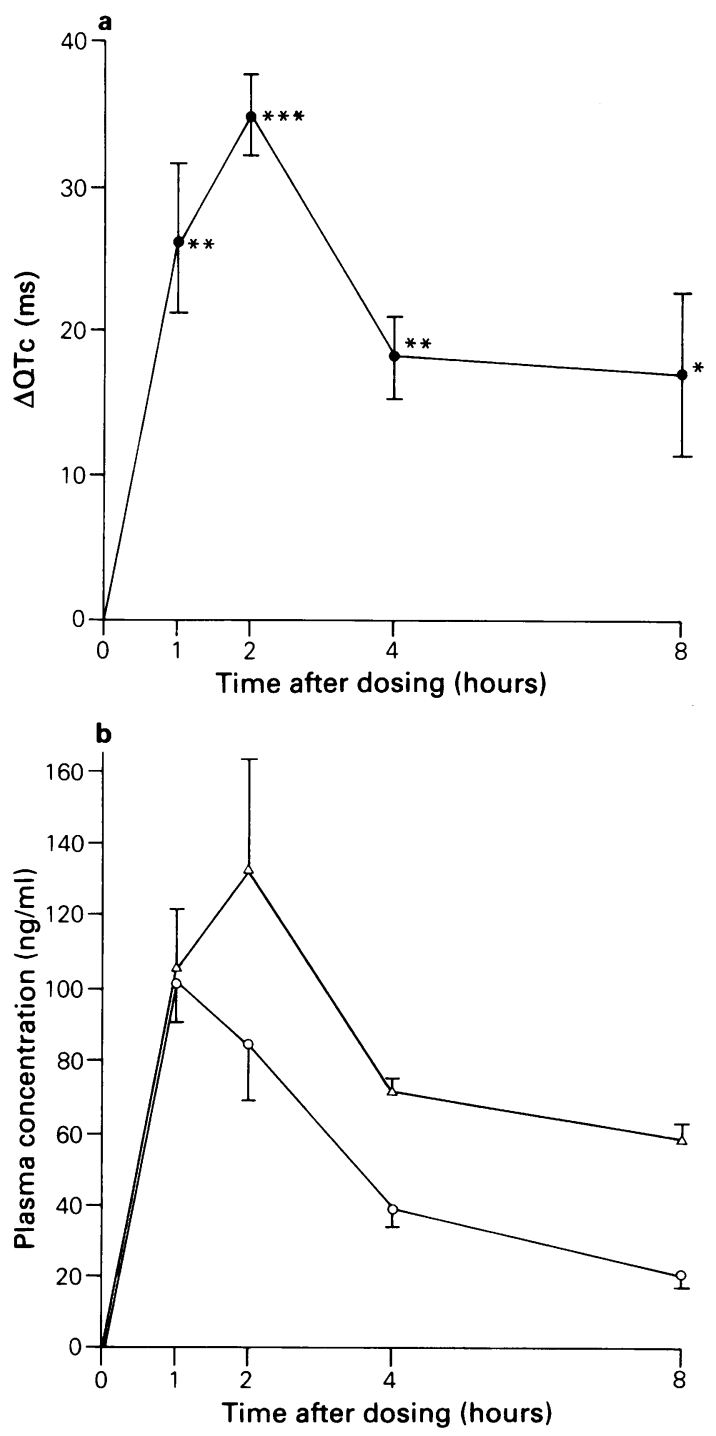

Figure 1 (a) Changes in $\mathrm{QT}_{\mathrm{c}}$ interval (ms) after a single $40 \mathrm{mg}$ dose of ketanserin (O). (b) Plasma concentrations of ketanserin $(O)$ and ketanserinol $(\Delta)$ after a single $40 \mathrm{mg}$ dose of ketanserin. Mean and s.e.m. data $(n=6)$. ${ }^{*} P<0.05 ;{ }^{* *} P<0.01 ;{ }^{* * *} P<0.001$. 


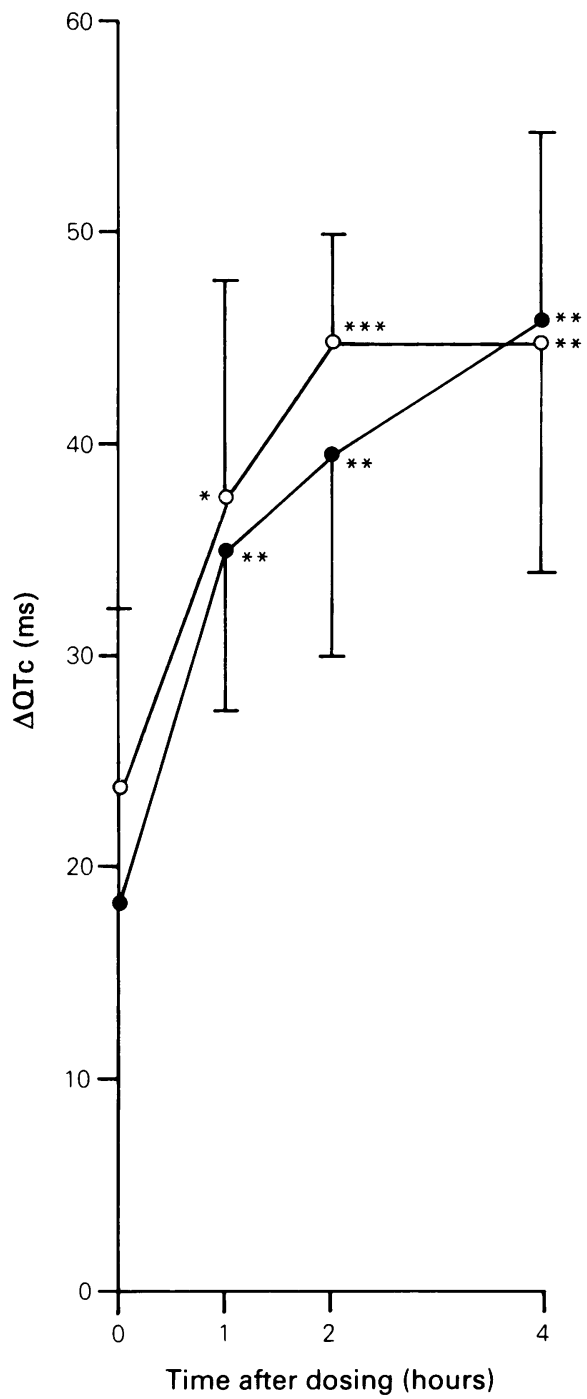

Figure 2 Changes in $\mathrm{QT}_{\mathrm{c}}$ interval (ms) from pretreatment values at steady-state during treatment with ketanserin $20 \mathrm{mg}$ b.d. (O) and $40 \mathrm{mg}$ b.d. (O). Mean and s.e.m. data $(n=6) . \quad * P<0.05 ; \quad * * P<0.01$ $* * * P<0.001$.

interval prolongation between pre-dose and 4-hour values. Changes in $\mathrm{QT}_{\mathrm{c}}$ interval were not clearly dose-dependent in this study, perhaps because of the small sample-size. They were only weakly correlated with plasma concentrations of ketanserin or ketanserinol, but followed a similar time-course to plasma drug and metabolite concentrations.

\section{Study 2}

Protocol Fifty-four hypertensive patients (33 men,
21 women, mean age 50.4 years) were randomly allocated to treatment with ketanserin $(n=26)$ at a mean daily dose of $73 \mathrm{mg}$ or to matching placebo $(n=28)$, each for 7 weeks. The $\mathrm{QT}_{\mathrm{c}}$ interval, plasma drug concentrations, serum potassium and serum calcium were measured at entry and at the final visit, 14 hours after dosing. The observations therefore correspond to the pre-dose 'trough' measurements in Study 1.

Results The mean $\mathrm{QT}_{\mathrm{c}}$ interval was similar in the two treatment groups at entry (ketanserin 420 , s.d. $23 \mathrm{~ms}$; placebo 410 , s.d. $27 \mathrm{~ms}$ ). Compared to placebo, ketanserin increased the $\mathrm{QT}_{\mathrm{c}}$ interval by $14 \mathrm{~ms}(95 \% \mathrm{CI}+4,+24 \mathrm{~ms}, P<0.01)$. The patients had been stratified into three sub-groups according to existing treatment (no treatment, bendrofluazide $5 \mathrm{mg} /$ day, or atenolol $100 \mathrm{mg} /$ day), and $\mathrm{QT}_{\mathrm{c}}$ interval prolongation was not significantly different whether ketanserin was used as monotherapy or added to a diuretic or $\beta$-blocker (mean increases $13.6 \mathrm{~ms}$, $9.1 \mathrm{~ms}$ and $20.3 \mathrm{~ms}$ respectively). In all ketanserintreated patients $(n=26)$ the change in $\mathrm{QT}_{\mathrm{c}}$ interval was negatively correlated with body weight $(r=-0.58, \quad P<0.01, \quad$ Figure 3$)$. $\mathrm{QT}_{\mathrm{c}}$ interval prolongation was observed particularly in women, who weighed less than men. After adjustment for weight, sex and age ketanserin still prolonged the $\mathrm{QT}_{\mathrm{c}}$ interval significantly compared to placebo, by a mean of $15 \mathrm{~ms}(95 \% \mathrm{CI}+5,+25 \mathrm{~ms}, P<0.01)$. The increase in $\mathrm{QT}_{\mathrm{c}}$ interval was significantly related to the dose of ketanserin when corrected for body weight $(r=0.63, P<0.01$, Figure 4$)$. There was no significant relation between plasma drug or metabolite concentrations and $\mathrm{QT}_{\mathrm{c}}$ prolongation. Serum potassium and calcium concentrations did not alter during the study and were not related to changes in the $\mathrm{QT}_{\mathrm{c}}$ interval.

The relation of uncorrected QT interval to $R-R$ interval was examined by linear regression to ensure that the findings described above were independent of Bazett's correction (see Methods). There was a significant difference of $22 \mathrm{~ms}(P<0.02)$ between the intercepts for ketanserin and placebo (Figure 5), indicating that ketanserin prolonged the QT interval by $22 \mathrm{~ms}$ independent of heart rate.

Electrocardiograms with $\mathrm{QT}_{\mathrm{c}}$ prolongation frequently showed $\mathrm{T}$ wave flattening, and in some cases the $T$ wave was bifid, independent of the $U$ wave (Figure 6). There was no change in QRS duration and thus QT interval prolongation resulted from lengthening of the JT segment.

Comment Ketanserin treatment prolonged the $\mathrm{QT}_{\mathrm{c}}$ interval significantly, by a mean of $14 \mathrm{~ms}$ when measured 14 hours after dosing. This pre-dose 


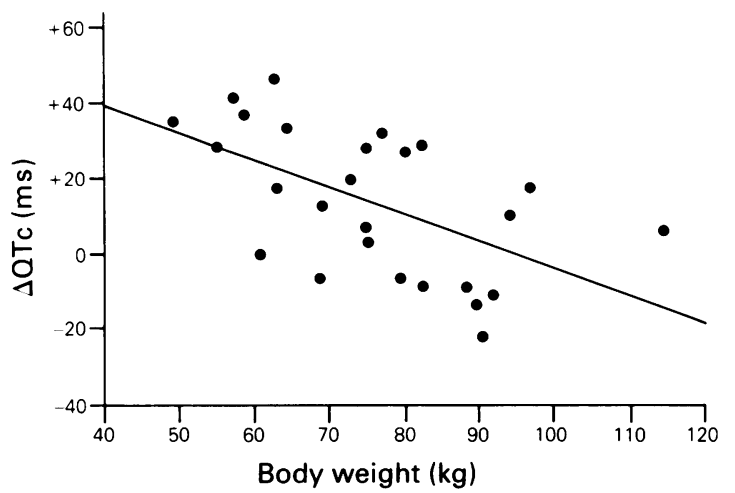

Figure 3 Relation of change in $\mathrm{QT}_{c}$ interval after ketanserin treatment and body weight $(n=26) . \Delta \mathrm{QT}_{\mathrm{c}}$ $=0.066-0.0007$ weight; $r=-0.58, P<0.01$.

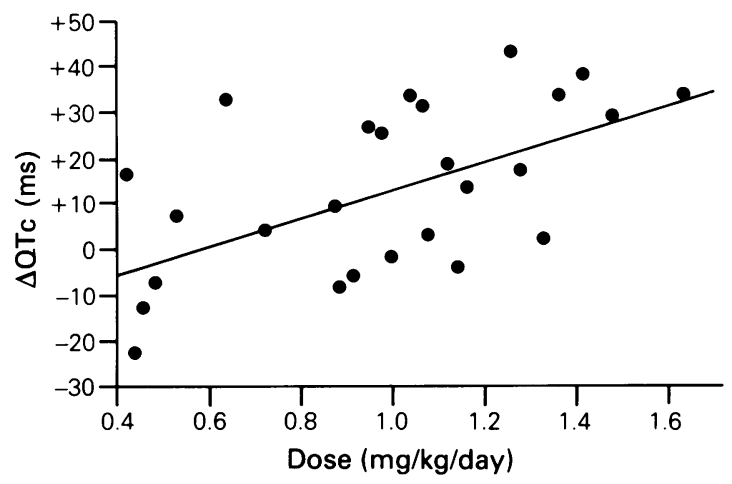

Figure 4 Relation of $\mathrm{QT}_{\mathrm{c}}$ interval and dose of ketanserin $(n=26) . \Delta \mathrm{QT}_{\mathrm{c}}=0.0607$ dose $-0.017 ; r=0.63$, $P<0.01$.

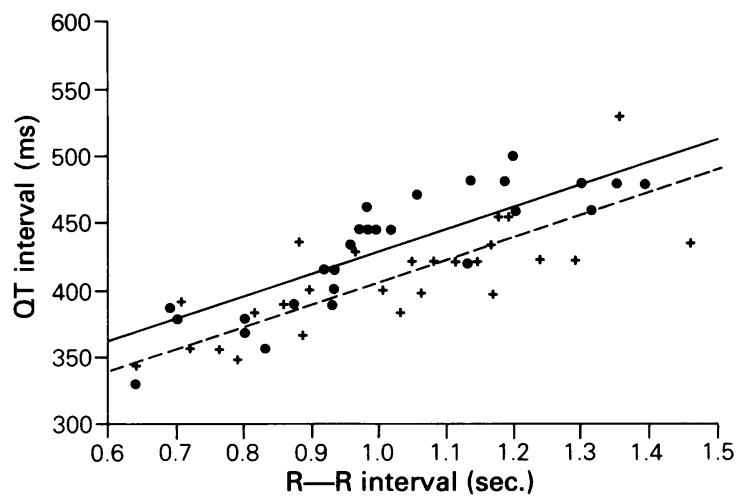

Figure 5 Relation of uncorrected QT and R-R intervals after parallel constraint during treatment with ketanserin $(n=26)$ and placebo $(n=28)$. Ketanserin: - $; \mathrm{QT}=0.262+0.165 \mathrm{R}-\mathrm{R}$. Placebo: +----; $\mathrm{QT}=0.240+0.165 \mathrm{R}-\mathrm{R}$. Difference between intercepts $=0.22 \mathrm{~ms} . P<0.02$. increase is likely to be doubled 4 hours after dosing, as shown in Study $1 . \mathrm{QT}_{\mathrm{c}}$ prolongation was related to body weight, and the regression equation predicts substantial prolongation ( $30 \mathrm{~ms}$ or more) in those weighing $50-60 \mathrm{~kg}$, but no increase in those weighing $90-100 \mathrm{~kg}$. Lengthening of the $\mathrm{QT}_{\mathrm{c}}$ interval with ketanserin was dose-dependent. A dose of $1.6 \mathrm{mg} / \mathrm{kg} /$ day increased the $\mathrm{QT}_{\mathrm{c}}$ interval by an average of $30 \mathrm{~ms} 14$ hours after dosing, whereas a dose of $0.6 \mathrm{mg} / \mathrm{kg} /$ day caused no change. Increases in the $\mathrm{QT}_{\mathrm{c}}$ were unrelated to concentrations of ketanserin, ketanserinol, potassium or calcium in blood.

\section{Study 3}

Protocol Twenty-one patients with intermittent claudication (14 men, 7 women, mean age 59.8 years) were randomly allocated to ketanserin, mean dose $167 \mathrm{mg} /$ day or placebo, each given for 3 months. The $\mathrm{QT}_{\mathrm{c}}$ interval was measured 2-4 hours after dosing.

Results The two treatment groups were wellmatched at entry as regards $\mathrm{QT}_{c}$ interval (ketanserin 412, s.d. $39 \mathrm{~ms}$; placebo, 408, s.d. $42 \mathrm{~ms})$. The mean increase in $\mathrm{QT}_{c}$ interval with ketanserin, compared to placebo was $40 \mathrm{~ms}(95 \%$ $\mathrm{CI}+7,+73 \mathrm{~ms}, P<0.001)$. The largest individual increase observed was $80 \mathrm{~ms}$.

Comment These data confirm marked prolongation of the $\mathrm{QT}_{c}$ interval with high doses of ketanserin. They also show that the effect is persistent for at least three months.

\section{Discussion}

These studies confirm a previous report $^{6}$ that ketanserin prolongs the $\mathrm{QT}_{\mathrm{c}}$ interval. Substantial $\mathrm{QT}_{\mathrm{c}}$ prolongation was observed after a single oral dose of $40 \mathrm{mg}$, and it became more marked during chronic dosing with $20 \mathrm{mg}$ or $40 \mathrm{mg}$ ketanserin twice daily. Prolongation of the $\mathrm{QT}_{c}$ varied markedly during the dose interval, with maximum increases four hours after dosing which were approximately double those observed at the end of a dose interval. Prolongation of the $\mathrm{QT}_{\mathrm{c}}$ interval was related to the dose of ketanserin corrected for body weight. It was not related, or at most weakly related, to plasma concentrations of ketanserin or its metabolite ketanserinol, a finding that contrasts with observations made during treatment with amiodarone ${ }^{11}$ and sotalol. ${ }^{12}$ Lengthening of the $\mathrm{QT}_{\mathrm{c}}$ interval was associated with $\mathrm{T}$ wave flattening 

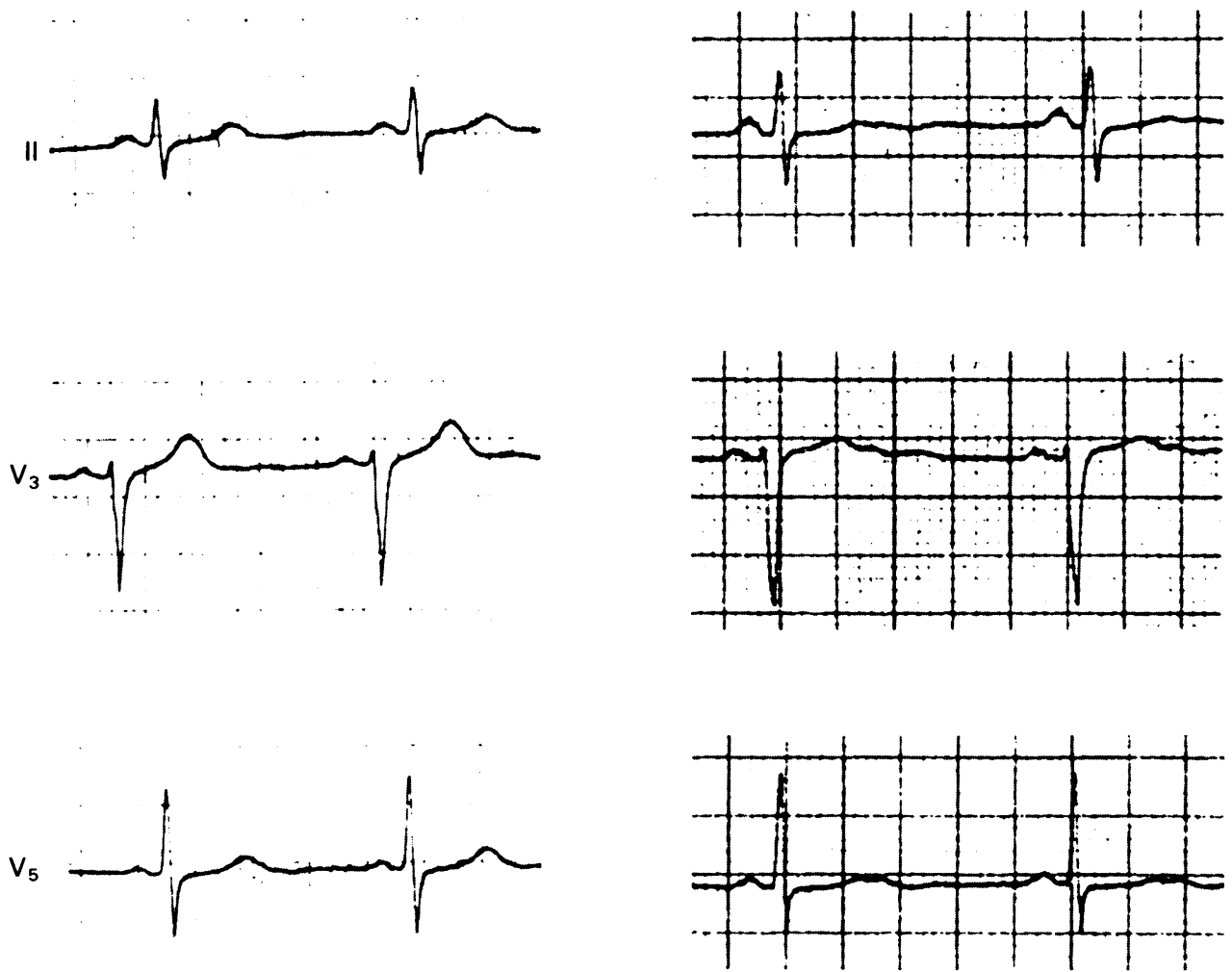

Figure 6 Electrocardiograms taken before and during treatment with ketanserin, showing prolongation of the QT interval. Note that this is independent of the $U$ wave.

and changes in $T$ wave morphology similar to those reported with amiodarone. ${ }^{11}$ Ketanserin-induced prolongation of the $\mathrm{QT}_{\mathrm{c}}$ was observed when the drug was prescribed as monotherapy, and when it was added to thiazide diuretic or $\beta$-blocker therapy. It was not related to serum potassium or calcium concentrations. Extreme $\mathrm{QT}_{\mathrm{c}}$ prolongation, up to $80 \mathrm{~ms}$, was observed at high doses of ketanserin.

Drugs which prolong the QT interval substantially may predispose patients to serious cardiac arrhythmias, particularly the form of ventricular tachycardia known as torsade de pointes. ${ }^{7}$ Ketanserin may be introduced for the treatment of hypertension in the near future, and the dose proposed is $40-80 \mathrm{mg} /$ day. The results presented here suggest that a dose of $80 \mathrm{mg} / \mathrm{day}$ would prolong the $\mathrm{QT}_{\mathrm{c}}$ interval by an average of $30 \mathrm{~ms}$ in a patient weighing $50 \mathrm{~kg}$ when it is measured 14 hours after dosing. In such a patient the expected increase four hours after dosing would be around $60 \mathrm{~ms}$. We have not observed arrhythmias during ketanserin treatment, but as far as we are aware all drugs which prolong the QT interval to this extent have been associated with serious arrhythmias. Four cases of ventricular arrhythmias during treatment with ketanserin have already been reported. ${ }^{13,14}$ If ketanserin does become available for use in hypertension it will be prescribed to patients with a high prevalence of heart disease, and it may be prescribed with other drugs which prolong the QT interval. The risk of arrhythmias needs to be quantitated in a large population of patients before it comes into general use. Meanwhile, patients treated with ketanserin should have their QT interval monitored regularly, and should not be treated with other drugs known to prolong the QT interval.

\section{Acknowledgements}

Financial support for H.A.C. and P.C.W. from Janssen Pharmaceutical Ltd is gratefully acknowledged. We thank Dr G.T. Tucker for analysis of plasma drug concentrations, L. Parnell and C. Cameron for technical assistance and $\mathrm{A}$. Whitehead for statistical advice. 


\section{References}

1. McGourty, J.C., Silas, J.H. \& Cowen, K.J. Controlled trial of ketanserin in hypertension. $\mathrm{Br} J$ Clin Pharmacol 1985, 20: 37-40.

2. Cameron, H.A. \& Ramsay, L.E. Ketanserin in essential hypertension: a double-blind, placebocontrolled study. Postgrad Med J 1985, 61: 583-586.

3. DeCree, J., Leempoels, J., Geuken, H. \& Verhagen, H. Placebo-controlled double-blind trial of ketanserin in treatment of intermittent claudication. Lancet 1984, ii: 775-779.

4. Bounameaux, H., Holditch, T., Hellemans, H., Berent, A. \& Verhaeghe, R. Placebo-controlled, double-blind, two centre trial of ketanserin in . intermittent claudication. Lancet 1985, ii: 1268-1271.

5. Stranden, E., Roald, O.K. \& Krohg, K. Treatment of Raynaud's phenomenon with the 5-HT 2 receptor antagonist ketanserin. $\mathrm{Br}$ Med J 1982, 285: 10691071.

6. Stott, D.J., McClenachan, J.M. \& Ball, S.G. Ketanserin, the QT interval, and autonomic function testing in normal subjects. Br J Clin Pharmacol 1985, 20: 84P (abstract).

7. Soffer, J., Dreifus, L.S. \& Michelson, E.L. Polymorphous ventricular tachycardia associated with normal and long QT intervals. Am J Cardiol 1982, 49: 20212029.
8. Bazett, H.C. An analysis of the time-relations of electrocardiograms. Heart 1920, 7: 353-370.

9. Staniforth, D.H. The QT intervals and cycle length: the influence of atropine, hyoscine and exercise. $B r J$ Clin Pharmacol 1983, 16: 615-621.

10. Snedecor, G.W. \& Cochran, W.G. Statistical Methods, 6th edition. Iowa State University Press, Iowa, 1967, pp 432-436.

11. Debbas, N.M.G., du Cailar, C., Bexton, R.S., Demaille, J.G., Camm, A.J. \& Puech, P. The QT interval: a predictor of the plasma and myocardial concentrations of amiodarone. Br Heart $J$ 1984, 51: 316-320.

12. Neuvonen, P.J., Elonen, E., Tanskanen, A. \& Tuomilento, J. Sotalol and prolonged QT interval, Lancet 1981, ii: 426 (letter).

13. Verstraete, M. Drugs acting on the peripheral circulation. In: Side-effects of Drugs Annual 8. Elsevier, Amsterdam, 1984, pp 199-201.

14. Bajan, A., Aldariz, A.E., Baglino, H. et al. QTprolongacion del QT por ketanserin e inducion de arritmias ventriculares malginas. Rev Argent Cardiol 1985, 53: 588-593. 